\title{
The State of Peptide Receptor Radionuclide Therapy and Its Sequencing among Current Therapeutic Options for Gastroenteropancreatic Neuroendocrine Tumors
}

\author{
Lauren M. Raymond ${ }^{a}$ Tetiana Korzun ${ }^{a}$ Adel Kardosh $^{a, b}$ Kenneth J. Kolbeck ${ }^{a, c}$ \\ Rodney Pommier ${ }^{a, d}$ Erik S. Mittra ${ }^{a, e}$ \\ ${ }^{a}$ School of Medicine, Oregon Health \& Science University, Portland, OR, USA; ${ }^{b}$ Division of Hematology/Medical \\ Oncology, Department of Internal Medicine, Oregon Health \& Science University, Portland, OR, USA; 'Department \\ of Interventional Radiology, Oregon Health \& Science University, Portland, OR, USA; 'Division of Surgical Oncology, \\ Department of Surgery, Oregon Health \& Science University, Portland, OR, USA; 'Division of Nuclear Medicine \& \\ Molecular Imaging, Department of Diagnostic Radiology, Oregon Health \& Science University, Portland, OR, USA
}

\section{Keywords}

Gastroenteropancreatic neuroendocrine tumor .

Neuroendocrine tumors - Peptide receptor radionuclide therapy · Receptors · Peptide $\cdot{ }^{177}$ Lu-dodecanetetraacetic acid $^{0}-$ Tyr $^{3}$ ]octreotate

\begin{abstract}
Gastroenteropancreatic neuroendocrine tumors (GEP-NETs) are the most common form of neuroendocrine neoplasia, but there is no current consensus for the sequencing of approved therapies, particularly with respect to peptide receptor radionuclide therapy (PRRT). This comprehensive review evaluates the data supporting approved therapies for GEPNETs and recommendations for therapeutic sequencing with a focus on how PRRT currently fits within sequencing algorithms. The current recommendations for PRRT sequencing restrict its use to metastatic, inoperable, progressive midgut NETs; however, this may change with emerging data to suggest that PRRT might be beneficial as neoadjuvant therapy for inoperable tumors, is more tolerable than other treatment modalities following first-line standard dose somatostatin analogs, and can be used as salvage therapy after disease relapse following prior successful cycles of
\end{abstract}

PRRT. PRRT has also been shown to reduce tumor burden, improve quality of life, and prolong the time to disease progression in a broad spectrum of patients with GEP-NETs. As the various potential benefits of PRRT in GEP-NET therapy continues to expand, it is necessary to review and critically evaluate our treatment algorithms for GEP-NETs.

(c) 2021 S. Karger AG, Basel

\section{Introduction}

Neuroendocrine neoplasms are a diverse group of epithelial malignancies arising from neuroendocrine cells throughout the body. Their site of origin leads to the heterogeneous nature of these tumors, and their ability to produce peptides leads to characteristic hormonal syndromes [1]. This review will focus on neuroendocrine neoplasms originating in the digestive tract and pancreas. Surgical resection is recognized as the only clinically curative measure for gastroenteropancreatic neuroendocrine tumors (GEP-NETs); however, given that these tumors have a propensity to metastasize and the primary tumor may, in some cases, be unresectable, various treatment options have been introduced including cytoreduc- 


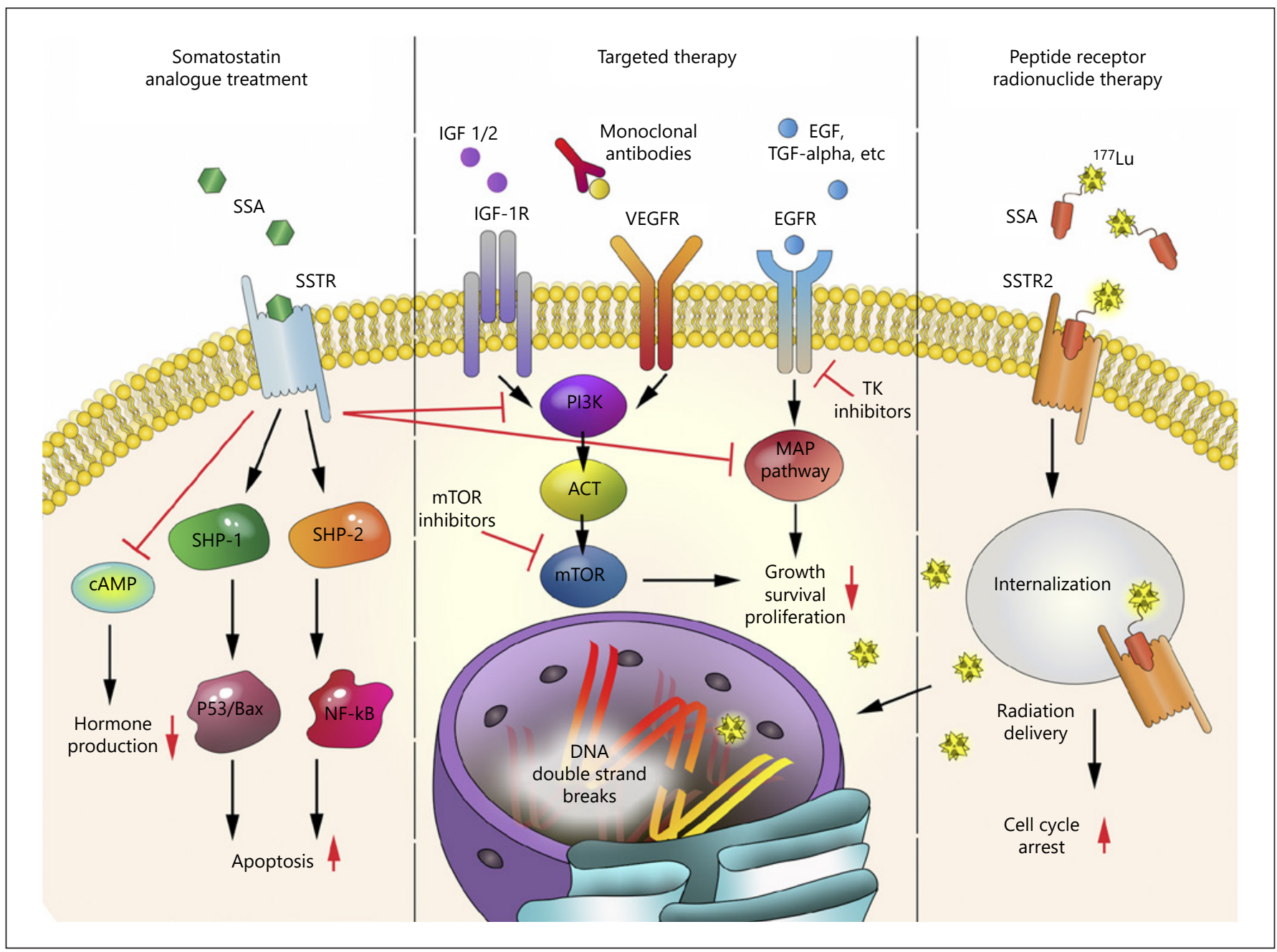

Fig. 1. Treatment options for GEP-NETs. An overview of treatment options and affected pathways in GEP-NETs. SSA, somatostatin analog; SSTR, somatostatin receptor; cAMP, $3^{\prime}, 5^{\prime}$-cyclic adenosine monophosphate; SHP-1, Src homology region 2 domain-containing phosphatase-1; SHP-2, Src homology region 2 domain-containing phosphatase-2; Bax, Bcl-2-associated X protein; NF-kB, nuclear factor kappa-light-chain-enhancer of activated B cells; IGF 1/2, insulin-like growth factors 1/2; IGF-1R, insulin- like growth factor 1 receptor; VEGFR, vascular endothelial growth factor receptor; EGF, epidermal growth factor; TGF-alpha, transforming growth factor alpha; EGFR, epidermal growth factor receptor; PI3K, phosphoinositide 3-kinase; ACT, protein kinase B; mTOR, mammalian target of rapamycin; MAP pathway, mitogenactivated protein kinase pathway; 177Lu, lutetium-177; SSTR2, somatostatin receptor 2 . tive operations, catheter-based liver-directed treatment and needle ablation, somatostatin analogs (SSAs), mammalian target of rapamycin (mTOR) inhibitors, tyrosine kinase inhibitors, systemic chemotherapy, and peptide receptor radionuclide therapy (PRRT) (shown in Fig. 1). Given the rise in incidence of GEP-NETs and the growing number of evidence-based therapies over the past decade, there is a heightened need for improved sequencing of these therapies to enhance the standard of care for patients with neuroendocrine tumors (NETs). 


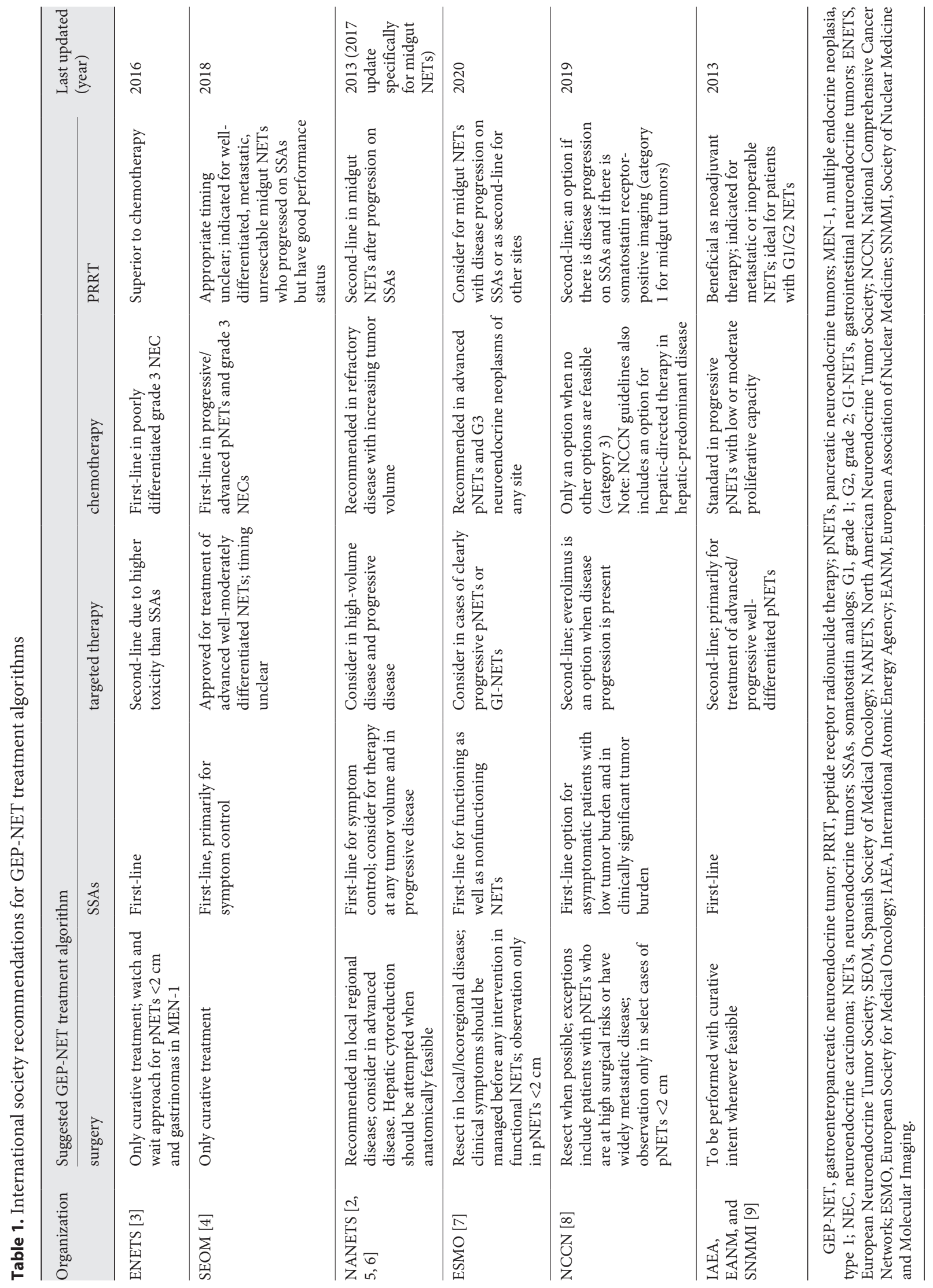


with the exception of the timing of PRRT. At this time, there is no global consensus as to the timing and sequencing of PRRT. With developing research on the widespread benefits of PRRT, as well as its limitations, it will be necessary to reevaluate current sequencing guidelines through a lens that encompasses current and emerging data on PRRT to determine optimal patient care. In this narrative review, we examined original clinical research, case report studies, and narrative reviews in an effort to identify the current state of PRRT sequencing with respect to other treatment modalities for GEP-NETs, given the most current and up-to-date literature on GEP-NET therapy.

\section{Current Guidelines}

Based upon current guidelines as of December 2019 (Table 1), surgical resection is viewed as the only clinically curative treatment measure and should be considered whenever feasible. Hepatic cytoreductive surgery can be considered in liver-predominant disease [5]. If the patient is not a surgical candidate, needle ablation and/or catheter-based liver-directed treatment can be considered for liver metastases in liver-predominant disease [7]. SSAs are used first-line for symptom control and have demonstrated antiproliferative properties to prolong time to tumor progression. Targeted therapies follow SSAs as a promising second-line therapy, and chemotherapy is utilized in a smaller subset of patients with advanced disease. The timing of PRRT, however, is uncertain. More clinically focused organizations including the Spanish Society of Medical Oncology (SEOM), the North American Neuroendocrine Tumor Society (NANETS), the National Comprehensive Cancer Network (NCCN), and the European Society for Medical Oncology (ESMO) state that PRRT is an option for well-differentiated to moderately differentiated, metastatic, unresectable midgut NETs that demonstrated disease progression on SSAs $[2,4,6,8,9]$. The European Neuroendocrine Tumor Society (ENETS) notes that PRRT is superior to chemotherapy but does not attempt to place PRRT within a treatment algorithm [7]. In contrast, research-focused organizations including the International Atomic Energy Agency (IAEA), European Association of Nuclear Medicine (EANM), and Society of Nuclear Medicine and Molecular Imaging (SNMMI) suggest that PRRT might be beneficial as neoadjuvant therapy to reduce tumor burden and allow for future resection [3]. The following describes the rationale and data to support each current treatment modality for GEP-NETs.

PRRT and Its Sequencing for GEP-NETs

\section{Existing Therapies}

\section{Surgery}

Complete surgical resection of the primary tumor, nodal metastases, and, in some cases, all visible metastatic disease is the only treatment modality that offers clinical cure. However, this can be accomplished in a minority of patients. The majority of patients with GEP-NETs present with liver metastases, which are usually bilobar and often numerous. The primary tumor may be small and elude detection by imaging or endoscopy and therefore be declared as "occult."

The vast majority of "occult" primary tumors can be located by surgical exploration, which can even be done laparoscopically $[10,11]$. This, at the very least, can guide systemic treatments and define eligibility for various clinical trials. There have been several studies that have shown improved survival with resection of the primary gastrointestinal neuroendocrine tumors (GI-NETs) in the setting of liver metastases [11-13]. Additionally, there is emerging evidence to suggest that resection of both gastrointestinal and pancreatic NET primaries may improve survival irrespective of whether or not the liver disease is treated [14]. Given the propensity for NETs to metastasize, another goal of surgical intervention is to thoroughly stage the patient through evaluation of surrounding structures as well as the liver, as a significant percentage of patients who do not have liver metastases apparent on preoperative imaging will have them found at the time of surgical exploration [5].

Eligibility for liver operations is considerably different for patients with metastatic NETs than from those with other types of cancer. Typically, hepatic resections are not performed unless a complete resection of the tumor can be accomplished and there is no extrahepatic disease. This is based on outcomes data showing that incomplete resection and positive margins lead to tumor recurrence and unfavorable patient outcomes. However, in hepatic NET metastases, the tumors are not infiltrative, making it possible to separate the tumor from the surrounding liver tissue and enucleate the tumors while sparing liver parenchyma. Therefore, significant liver debulking can achieve equivalent benefits in NET metastases as a complete resection would in other cancers [11,15-18].

Based upon a study by McEntee et al. [15], a consensus workshop in Berlin in 1996 determined that cytoreductive operations should be considered if $>90 \%$ of the imaged tumor volume in the liver can be safely removed and an operative mortality of $<10 \%$ can be ensured; this has been supported by several published series [19]. A more 
Table 2. Phase III clinical trials for GEP-NET treatment

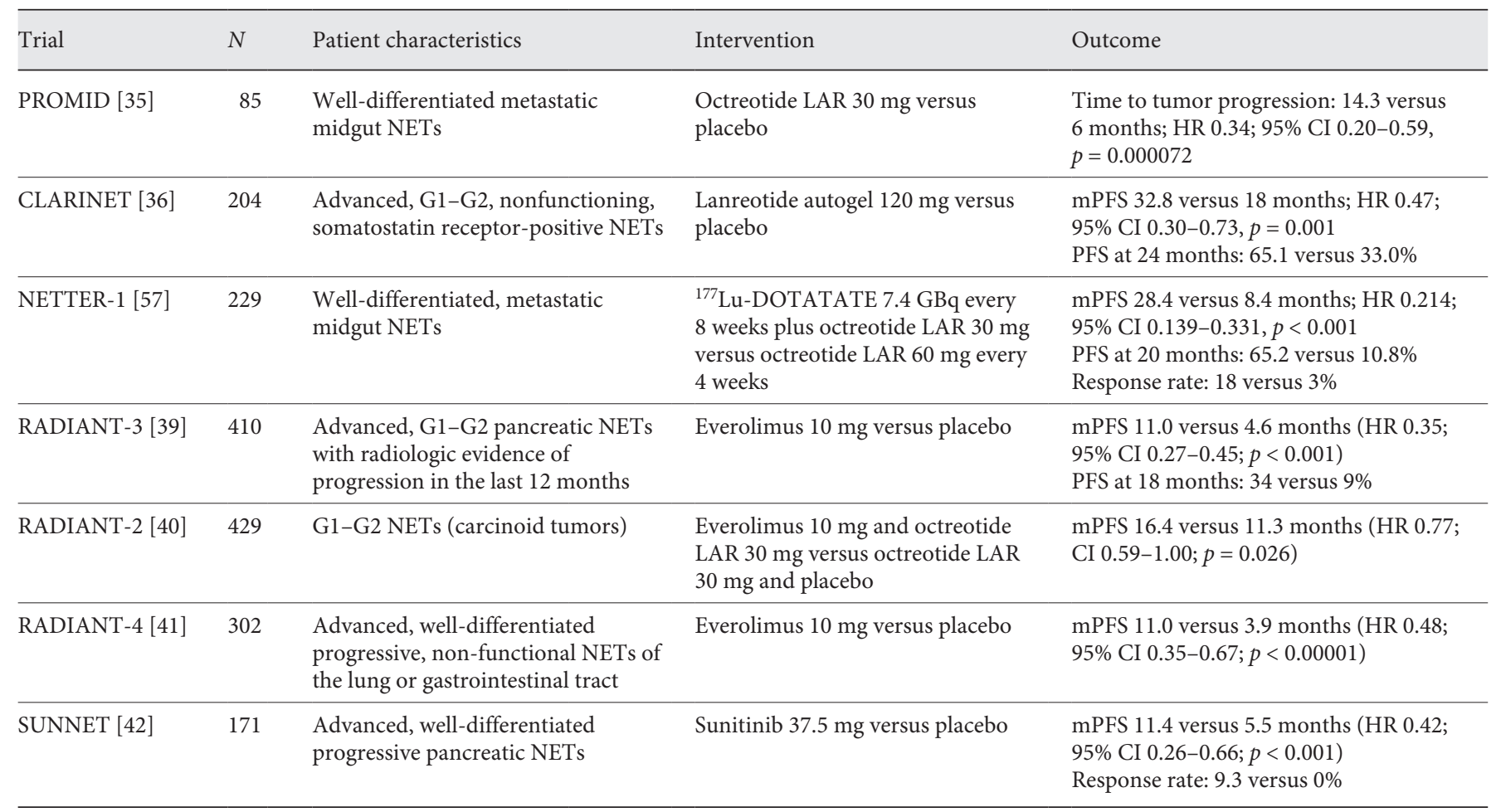

$N$, total number of patients in the trial; NETs, neuroendocrine tumors; LAR, long-acting release; HR, hazard ratio; CI, confidence interval; G1-G2, grade 1 to grade 2; mPFS, median progression-free survival; PFS, progression-free survival; ${ }^{177} \mathrm{Lu}$-dodecanetetraacetic acid ${ }^{0}$-Tyr ${ }^{3}$; CLARINET, study of lanreotide autogel in non-functioning entero-pancreatic endocrine tumours.

recent study from 2014 expanded the eligibility criteria for GI-NETs to include a liver tumor debulking threshold of $70 \%$, allowing positive resection margins by tumor enucleation, and allowing extrahepatic disease. The 5 -year disease-specific survival was $90 \%$ and the median time to liver progression was 71.6 months $[11,20]$. All deaths were from liver failure and no patient died of extrahepatic disease. A subsequent series in 2016 showed that these expanded criteria could also be extended to patients with pancreatic NETs (pNETs) with similar outcomes [21]. The 70\% debulking threshold was further supported for both GI-NETs and pNETs in a series from Iowa [22]. The utility of hepatic cytoreductive operations is reflected in the 2017 NANETS small bowel NET guidelines, where it is noted that cytoreduction should be attempted when anatomically feasible, even when the entire extent of liver metastases cannot be removed [5]. A similar recommendation for patients with pNETs was also published in the 2020 NANETS guidelines [23].

Some patients who may not be candidates for surgical cytoreduction at diagnosis may be rendered surgical can- didates following neoadjuvant therapies. Neoadjuvant oral chemotherapy with capecitabine and temozolomide has been successfully used for patients with metastatic pNETs, and PRRT has also been used in one series of patients with marginally operable pNETs with some success $[24,25]$. A particularly interesting finding in the latter series was a reduced incidence of subsequent liver metastases.

\section{Interventional Radiology}

Techniques including needle ablation and liver-directed catheter-based treatments have been implemented to control symptoms and improve survival outcomes in liver-predominant, inoperable metastatic disease. With supportive care alone, the 5-year survival of patients with unresectable liver metastases is $0-22 \%$ [26-28]. With the implementation of liver-directed embolotherapy, this 5 -year survival rate has increased to over $60 \%$, with combined biochemical and objective response rates between 70 and $90 \%$ [29]. The success of the interventional radiology-based therapeutic approach is at least in part due to 
the fact that NETs tend to form highly vascular metastatic lesions in the liver. The hepatic arterial supply to the lesion can be targeted with embolic agents (creating an ischemic environment near the lesion), chemoembolic agents (combining chemotherapy with decreased blood flow), and yttrium-90 $\left({ }^{90} \mathrm{Y}\right)$ radioembolization (selective internal radiation) [28].

According to the most recent ENETS guidelines, needle ablative techniques including radiofrequency ablation, microwave ablation, cryoablation, and irreversible electroporation are commonly used for liver metastases that are $<5 \mathrm{~cm}$, whereas larger or innumerable metastases can be treated with catheter-based arterial treatments [7]. This recommendation is largely because needle ablative techniques target specific lesions for each treatment, which can result in numerous individual needle ablations in a patient with several liver metastases. Therefore, this subset of techniques is restricted to patients with smaller and/or fewer liver metastases.

In contrast to needle ablative techniques, catheterbased liver-directed therapies consist of multiple distinct techniques including bland embolization which uses embolic particles targeted for the hepatic artery to cut off tumor blood supply, classical transarterial chemoembolization which uses an ethiodized oil emulsion of chemotherapeutic drugs, drug-eluting bead transarterial chemoembolization which uses drugs loaded onto embolic microspheres, and radioembolization which uses 30- to 60 -micron beads labeled with ${ }^{90} \mathrm{Y}$ to deliver radiation directly into the hepatic artery $[28,29]$. In regard to bland embolization versus chemoembolization, there appears to be no significant difference in outcomes with a 5-year survival of $50-65 \%$ in chemoembolization in comparison to $40-67 \%$ in bland embolization $[28,30]$. In order to elucidate an optimal treatment algorithm for NET liver metastases, the RETNET trial, which is an active, prospective, randomized controlled trial, is comparing bland embolization to classical transarterial chemoembolization with a primary endpoint of hepatic progression-free survival (PFS) [29]. This study initially included a drug-eluting bead transarterial chemoembolization arm as well, but this therapy was removed from the trial secondary to patient complications. The primary limitation to these techniques is that the treatment response may be incomplete because the periphery of the tumor may be spared from ischemia or chemotherapy. Therefore, patients with large, bulky hepatic tumor burden and relatively wellpreserved liver function are frequently treated with radioembolization due to the limited side effects, whereas patients with smaller tumor burden or small vascular distri- butions are treated with chemoembolic techniques [31]. In terms of therapeutic sequencing, it is also important to take into account cumulative radiation exposure when deciding on a specific treatment plan for a patient. For example, there is a risk of hepatotoxicity or hepatic failure if radioembolization is used in combination with PRRT [32].

\section{Somatostatin Analogs}

Patients with NETs often experience symptoms related to the overexpression of hormones from the neuroendocrine cells that have become neoplastic. Common symptoms include flushing, diarrhea, nausea, and vomiting. Somatostatin is a peptide hormone that acts as an inhibitor in many endocrine systems including endocrine and exocrine secretion, angiogenesis, cell proliferation, and initiation of apoptosis in some conditions [33]. Given that the half-life of native somatostatin is only 1-3 min and it is impractical to obtain for clinical use, synthetic derivatives of somatostatin have been developed to resist enzymatic degradation, extending the half-life of this synthetic hormone for sustained therapeutic use. Synthetic somatostatin derivatives were also created with the intent of retaining the capacity to bind to somatostatin receptors that are commonly found on tumoral tissue, allowing for the stable compound to be taken up by these receptors in order to generate their inhibitory effects [34]. The 2 primary commercially available SSAs are octreotide (Sandostatin, Novartis Pharmaceuticals Corporation) and lanreotide (Somatuline, Ipsen Biopharmaceuticals). Octreotide is available as a short-acting subcutaneous injection or a monthly long-acting repeatable (LAR) injection given every 28 days, whereas lanreotide is only available as an injectable autogel, given as a deep subcutaneous injection every 28 days. Prior to the "Study to Investigate the Antiproliferative Effect of Octreotide in Patients with Metastasized Neuroendocrine Tumors of the Midgut" (PROMID), which was a prospective, multicenter, randomized, double-blind, placebo-controlled trial in patients with well-differentiated metastatic midgut NETs, SSAs were used to treat the symptoms of patients with functionally active NETs but were not considered therapeutic for the treatment of the tumor itself.

The PROMID trial demonstrated that octreotide LAR would in fact prolong time to tumor progression versus placebo (Table 2). Subgroup analyses suggested that the antiproliferative effect of octreotide LAR was influenced by hepatic tumor burden, time since diagnosis, and whether or not the primary tumor had been resected [35]. Following the PROMID trial, the "Study of Lanreotide 
Autogel in Non-functioning Entero-pancreatic Endocrine Tumours" (CLARINET) demonstrated that lanreotide could also serve as a therapeutic modality to improve PFS in patients with advanced NETs. The CLARINET trial was a prospective, multicenter, international, randomized, double-blind, placebo-controlled study of lanreotide autogel (extended-release aqueous gel formulation) in patients with advanced, well-differentiated or moderately differentiated, nonfunctioning, somatostatin receptor-positive NETs who had documented disease progression. It should be noted that $96 \%$ of patients enrolled in the trial had stable disease in the 3- to 6-month period prior to randomization. Despite this, lanreotide was associated with a prolonged PFS of 32.8 versus 18.0 months in the placebo arm $(p=0.001)$ [36]. The openlabel extension, which comprised 89 patients (lanreotidelanreotide 42 ; placebo-lanreotide 47 ) with $38 \%$ pancreatic, $38 \%$ midgut, and $24 \%$ hindgut/unknown origin NETs, allowed patients with stable disease in the core study to continue lanreotide or switch from placebo to lanreotide in the open-label extension. The results were suggestive of continued antitumor effects, regardless of tumor origin [37].

\section{Targeted Therapy}

Targeted therapy for GEP-NETs is a broad umbrella that encompasses several different mechanisms of tumor suppression. Based upon available randomized controlled trial data, the primary focus of targeted therapy in the treatment of GEP-NETs involves inhibition of the mTOR pathway and tyrosine kinase pathway.

Neuroendocrine tumorigenesis has in part been linked to altered signaling in the mTOR pathway, and due to limited treatments for advanced NETs, the mTOR pathway continues to be a therapeutic target. The mTOR multiprotein complexes, mTORC1 and mTORC2, serve as intracellular serine-threonine kinase and are involved with key regulatory functions including cell growth, proliferation, angiogenesis, and metabolism [38]. An oral inhibitor of mTOR, everolimus (RAD001, Afinitor, Novartis Pharmaceuticals Corporation), was utilized in several RADIANT trials to demonstrate the significant antiproliferative effects of targeted therapy on NETs (Table 2) [39-41].

Although the RADIANT trials determined that there was an increased PFS benefit in patients with NETs treated with everolimus compared to a placebo, the outcome was similar in those who were treated with SSAs alone. When considering mTOR inhibitors as first-line therapy for GEP-NETs, everolimus tends to fall short due to its comparable outcomes with SSAs and its considerably worse side effects. The RADIANT- 2 trial in particular reported serious adverse events in $57 \%$ of the patients in the everolimus arm and $35 \%$ in the control arm, with the most commonly reported serious adverse events including diarrhea, interstitial lung disease, and thrombocytopenia. Although the adverse drug events noted in these trials were consistent with the manufacturer's safety profile, everolimus did produce significant side effects when compared with first-line SSA therapy.

Targeted therapy for GEP-NETs also includes the multitargeted tyrosine kinase inhibitor sunitinib malate (Sutent, Pfizer). Malignant pNETs have been shown to produce widespread expression of platelet-derived growth factor receptors, stem-cell factor receptor (c-kit), and vascular endothelial growth factor receptors. Sunitinib malate is a multikinase inhibitor that is able to target these receptors and delay tumor growth by reducing endothelial cell density and pericyte coverage of tumor vessels. The "Study of Sunitinib Compared to Placebo for Patients with Advanced Pancreatic Islet Cell Tumors" (SUNNET), a prospective, multicenter, international, randomized, double-blind, placebo-controlled study in patients with advanced, well-differentiated pancreatic NETs with documented disease progression within 12 months of enrollment, demonstrated an increased mPFS in patients treated with sunitinib of 11.4 months versus placebo of 5.5 months $(p<0.001)$. The objective response rate was $9.3 \%$ in the sunitinib group versus $0 \%$ in the placebo group (Table 2) [42].

\section{Systemic Therapy}

Chemotherapy is currently recommended for poorly differentiated grade-3 (G3) GEP-NECs and in progressive or advanced pNETs. Overall survival is poor in NECs, ranging from 5 months in metastatic disease to 38 months in patients with localized disease [43]. In patients with metastatic NECs, survival varies widely with chemotherapy (7-19 months) but still shows a substantial improvement over patients who receive only best supportive care (1 month) [43]. In GEP-NECs, a platin and etoposide regimen has been the most widely used regimen with response rates of $30 \%$ and median survival of around 12 months [43-45]. Historically, for pNETs, chemotherapy regimens included streptozocin with either 5-fluorouracil (5-FU) or doxorubicin. Other active agents included dacarbazine or temozolomide [8]. Specifically among pNETs, streptozocin with 5-FU had a PFS of 16-23 months and an overall response rate of $28-42 \%$ [46]. When streptozocin, 5-FU, and doxorubicin were com- 
bined, the PFS was 18 months with a median overall survival (mOS) of 37 months, which is comparable to streptozocin and 5-FU alone [47]. Although a number of chemotherapy regimens have been approved for use in patients with pNETs, due to their significant side effect profiles many of these regimens are not used in the clinical setting. More recently, temozolomide and capecitabine have been studied as first-line therapy for pNETs with a mPFS of 18 months and an objective response rate of $71 \%$ [48]. This regimen is now used clinically as it is more effective than historical chemotherapy regimens and produces fewer side effects.

\section{Peptide Receptor Radionuclide Therapy}

PRRT uses radiolabeled SSAs to irradiate the neoplastic cells in NETs. Given that functioning NETs secrete biologically active hormones, PRRT was developed as a way to take advantage of the active hormone, which would be localized to and internalized by these tumor cells with radioisotopes that emit radiation and can damage the neoplastic cells. The most known and studied SSA is octreotide, which has a high binding specificity to somatostatin receptor subtype 2 , the receptor expressed on NETs [49]. This peptide-receptor complex is then internalized into the cell and the associated isotope will irradiate the cell, causing DNA-damage and chromosomal aberrations. The 2 primary radioisotopes used for treatment of NETs are ${ }^{90} \mathrm{Y}$ and lutetium-177 $\left({ }^{177} \mathrm{Lu}\right) .{ }^{90} \mathrm{Y}$ is a pure high-energy beta-minus emitter with long-range particles that irradiates both the targeted and surrounding cells, while ${ }^{177} \mathrm{Lu}$ is a medium-energy beta-minus and gamma emitter. This suggests that ${ }^{90} \mathrm{Y}$ is potentially more useful for the irradiation of large masses, while ${ }^{177} \mathrm{Lu}$ allows for concentrated radiation to smaller masses [50]. According to most NET guidelines, PRRT is primarily used as a second-line therapy in patients with advanced or metastatic GEP-NETs. However, evolving data suggest that PRRT may have a broader spectrum of utility.

Prior to the "Study Comparing Treatment with ${ }^{177} \mathrm{Lu}$ DOTA $^{0}$-Tyr ${ }^{3}$-Octreotate to Octreotide LAR in Patients with Inoperable, Progressive, Somatostatin ReceptorPositive Midgut Carcinoid Tumours" (NETTER-1), several studies had demonstrated the efficacy of PRRT, but none had compared PRRT to existing therapy [51-56]. The NETTER-1 trial, a prospective, multicenter, international, randomized, double-blind, comparator-controlled study of $\left({ }^{177} \mathrm{Lu}\right.$-dodecanetetraacetic acid $\left.{ }^{0}-\mathrm{Tyr}^{3}\right)$ octreotate $\left({ }^{177} \mathrm{Lu}\right.$-DOTATATE) plus best-supportive care (octreotide LAR $30 \mathrm{mg}$ ) versus octreotide LAR $60 \mathrm{mg}$ alone in patients with well-differentiated, metastatic or locally advanced, inoperable midgut NETs, demonstrated that the addition of PRRT to first-line SSA therapy produced a statistically significant prolongation in PFS of 28.4 months in the ${ }^{177} \mathrm{Lu}$-DOTATATE plus octreotide LAR arm versus 8.4 months in the high-dose octreotide LAR alone arm $(p<0.001)$ [57]. Additionally, upon review by a blinded independent radiology committee, the response rate in the PRRT group was 13 versus $4 \%$ in the octreotide LAR group [58]. In the interim analysis of overall survival (OS), there were 14 deaths in the ${ }^{177} \mathrm{Lu}$ DOTATATE arm versus 26 in the control arm $(p=0.004)$. Regarding toxicity, a total of 201 patients (95\% in the ${ }^{177} \mathrm{Lu}$-DOTATATE group and $86 \%$ in the control group) experienced at least one adverse event; however, adverse events determined to be associated with treatment occurred in 129 patients. Patients that withdrew secondary to adverse events after the start of treatment included 7 patients $(6 \%)$ in the treatment group and 10 patients $(9 \%)$ in the control group. The majority of these events were considered grade 1 or 2 based upon the National Cancer Institute Common Terminology Criteria for Adverse Events (CTCAE) grading schema; however, grade 3 or 4 neutropenia, thrombocytopenia, and leukopenia were reported in 1,2 , and $9 \%$ of patients in the PRRT group versus no patients in the control group [57]. To date, NETTER-1 is the only randomized, controlled trial of PRRT for the treatment of NETs allowing for the US Food and Drug Administration (FDA) approval of ${ }^{177} \mathrm{Lu}-\mathrm{DOT}$ ATATE (Lutathera, Advanced Accelerator Applications, a Novartis company) in January 2018.

The side effects of PRRT are typically mild and often related to the coadministration of amino acids with the radiopeptide, which is performed to enlarge the safety margin when treating a patient with high-dose radiation [3]. The high-energy ${ }^{90} \mathrm{Y}$ is reported to have a higher incidence and severity of side effects than ${ }^{177} \mathrm{Lu}$, including adverse events such as myelosuppression and renal toxicity [59-61]. Since radiopeptide is reabsorbed in the proximal tubule of the kidney, subsequent radiopeptide retention leads to excessive radiation doses and renal failure. The effects of PRRT on kidney function was studied in 28 patients with metastatic NETs, which demonstrated a creatinine clearance loss of about $3.8 \%$ per year in patients treated with ${ }^{177} \mathrm{Lu}-\mathrm{DOTATATE}$ and $7.3 \%$ per year in patients treated with ${ }^{90} \mathrm{Y}$-dodecanetetraacetic acid ${ }^{0}$ $\mathrm{Phe}^{1}-\mathrm{Tyr}^{3}$ ) octreotide ( $\left.{ }^{90} \mathrm{Y}-\mathrm{DOT} A T O C\right)$ [59]. Additionally, the incidence of CTCAE grade 4 or 5 renal toxicity was $9.2 \%$ in patients treated with ${ }^{90} \mathrm{Y}$-DOTATOC [60]. To prevent this, positively charged amino acids are used to compete with the radiopeptide for reabsorption; how- 
ever, this leads to other side effects related to co-infusion, including nausea, headache, and rarely vomiting due to metabolic acidosis [61-63]. The severity of these side effects is related to the specific formulation of amino acids. In regard to bone marrow toxicity, CTCAE grade 3 and 4 acute bone marrow toxicity is observed in up to $13 \%$ of cases $[54,60]$. Sporadic cases of myelodysplastic syndrome and overt acute myelogenous leukemia have also been reported as possible long-term outcomes from PRRT [64].

\section{Emerging Research on the Utility of PRRT}

Following the NETTER-1 trial, researchers continue to study the utility and roles of PRRT (see online suppl. files 1-3; for all online suppl. material, see www.karger. com/doi/10.1159/000516015) [65]. One such postulated role is for PRRT to serve as neoadjuvant therapy in patients with inoperable GEP-NETs. In one specific study, 6 patients underwent treatment with ${ }^{90} \mathrm{Y}$-DOTATATE resulting in a mean reduction in tumor size of $1.5 \mathrm{~cm}$, allowing for 2 tumors to become surgically resectable [66]. Another study investigated if PRRT could be used interchangeably with SSAs as potential first-line therapy. This study concluded that PRRT in combination with SSAs improved mPFS by 21 months when compared to PRRT alone, suggesting that SSAs remain best practice as firstline therapy and should be used in combination with PRRT to achieve the best outcomes [67]. However, one case has been described in which PRRT was followed immediately after surgical resection and the patient was able to achieve a stable disease state with no ongoing symptoms. Although the patient is asymptomatic, at the time of publication, the patient's liver metastases had not responded to PRRT; therefore, it is difficult to determine if their stable disease status is due to the surgery or the PRRT [68].

Another area of study is combination or concurrent therapy, which investigates the effects of PRRT with other therapies, such as SSAs or chemotherapy. Given that SSAs are standard therapy for GEP-NETs and remain best-supportive care in the setting of disease progression, combination therapy involving SSAs is present even in clinical trials like NETTER-1. However, more research is now being conducted to focus on alternative combinations for the treatment of inoperable or metastatic disease, including PRRT in combination with chemotherapy. In a study that combined ${ }^{177} \mathrm{Lu}$-octreotate with capecitabine and temozolomide, patients with inoperable
NETs achieved a complete response rate of $15 \%$ with a mPFS of 31 months [69]. Additionally, there is at least one current clinical trial looking at the efficacy of targeted therapy in combination with PRRT, but no data have been published on the outcomes from this trial [70].

Another area of interest is salvage therapy after disease progression following initial treatment cycles of PRRT with or without additional prior therapies. As outlined in current guidelines, this is where treatment suggestions come to a halt. However, studies have shown that there may be some benefit to retreatment with PRRT following disease progression after prior successful cycles of PRRT [71-73]. Current data suggest that this may prolong PFS by up to $14-22$ months, leading to increased OS $[72,73]$.

Although PRRT has the potential to serve numerous roles in the treatment of GEP-NETs, direct sequencing studies are needed to determine where it fits best into the treatment algorithm for GEP-NETs. In one retrospective observational analysis of 1,182 patients in Italy, 4 main treatment algorithms were compared with emphasis on differences in mPFS. This analysis found that the mPFS was not statistically significant when SSAs were followed by high-dose SSA, targeted therapy, chemotherapy, or PRRT; however, the sequences with high-dose SSA and PRRT were better tolerated and thus less likely to be discontinued [74]. Likewise, a study of 611 patients with pNETs from the Netherlands Cancer Registry sought to determine the differences in OS based upon treatment type. In this study, surgical resection had the highest survival rates (86\%) followed by PRRT (33\%). Without surgery, PRRT was determined to have the best survival curves in patients with distant metastases [75]. The primary limitation to these studies is that they are retrospective observational studies and do not directly compare treatment algorithms in a prospective, randomized fashion, leading to the potential for a high risk of bias. However, these studies do allow for varying treatment considerations based upon the individual disease burden of each patient. Despite the present research on the roles of and possible utility of PRRT as neoadjuvant therapy, salvage therapy, or for concomitant use with other current GEPNET therapies, there are no current high-quality prospective studies that address PRRT sequencing.

\section{Ongoing and Upcoming Clinical Trials}

As of December 2019, PRRT remains an active area of research and head-to-head trials are beginning to emerge to determine how PRRT compares to other therapeutic modalities. Examples within this area of research include the "Efficacy and Safety of 177Lu-edotreotide PRRT in 
GEP-NET Patients" (COMPETE) and the "Antitumor Efficacy of Peptide Receptor Radionuclide Therapy with 177Lu-octreotate Randomized versus Sunitinib in Unresectable Progressive Well-differentiated Neuroendocrine Pancreatic Tumor: First Randomized Phase II" (OCCLURANDOM) trials. The COMPETE trial is a phase III prospective, multicenter, international, randomized, openlabel study that compares PRRT to everolimus in patients with inoperable, progressive GEP-NETs, with a focus on differences in PFS and OS [76]. The OCCLURANDOM trial is a phase II prospective, multicenter, randomized, open-label, comparator-controlled study that compares PRRT to sunitinib in unresectable, progressive, well-differentiated pNETs, with a primary outcome measure of differences in PFS at 12 months [77]. Additionally, the "Carcinoid Tumors After Failure of Somatostatin Analogs: a Randomized Phase III of Octreotide Lutate Peptide Receptor Radionuclide Therapy (PRRT) versus Interferon $a-2 b$ " (CASTOR) trial, a phase III prospective, multicenter, randomized, open-label comparison of PRRT to interferon alpha- $2 \mathrm{~b}$ in patients with progressive, unresectable, non-pancreatic GI-NETs resistant to SSA therapy, was a promising study but was ultimately withdrawn before the first patient was recruited to the trial [78]. In liver-predominant disease, there is also an ongoing phase II, prospective, non-randomized clinical trial comparing pembrolizumab in combination with liverdirected therapy (arterial embolization or cryoablation) to pembrolizumab in combination with PRRT with emphasis on evaluating the differences in overall response rates [79].

There are also a number of combination therapy trials that compare the efficacy of individual therapeutic treatments to options that combine PRRT with other approved therapies, including the "Capecitabine ON Temozolomide Radionuclide Therapy Octreotate Lutetium-177 NeuroEndocrine Tumours Study" (CONTROL NETS) and Lu-Ca-S trial. The CONTROL NETS trials are 2 parallel phase II trials that compare PRRT in combination with capecitabine (CAP)/temozolomide (TEM) chemotherapy to CAPTEM alone in patients with low to intermediate grade pNETs and to PRRT alone in patients with low to intermediate grade midgut NETs with a primary outcome of differences in PFS at 12 months for the pNET group and 24 months for the midgut NET group [80]. There is also another ongoing clinical trial separate from the CONTROL NETS trial that is investigating the PFS of CAPTEM chemotherapy in combination with PRRT in patients with advanced, inoperable G1/G2 GEP-NETs after 3 years [81]. Furthermore, the Lu-Ca-S trial, a phase
II study, also investigates capecitabine in combination with PRRT but randomizes the 2 study groups to either capecitabine in combination with PRRT followed by long-acting SSAs or PRRT alone followed by long-acting SSAs to evaluate differences in PFS at up to 72 months [82].

Clarifying and refining the treatment algorithm for GEP-NETs remains a strong area of research. Building upon the success of the NETTER-1 trial, the "Study to Evaluate the Efficacy and Safety of Lutathera in Patients with Grade 2 and Grade 3 Advanced GEP-NET" (NETTER-2) will evaluate the efficacy and safety of PRRT in combination with octreotide LAR compared to octreotide LAR alone in patients with G2/G3 advanced GEPNETs [83]. Furthermore, several other trials are investigating more broad applications of PRRT, including the treatment of other somatostatin receptor-positive tumors aside from GEP-NETs.

\section{Conclusion}

This narrative review highlights the fact that the current approval for PRRT, for metastatic, inoperable, progressive GEP-NETs, is generally well-suited but also open-ended. The latter allows for the utilization of PRRT in a variety of situations but also currently results in ambiguity with respect to sequencing this therapy. As has been highlighted, PRRT is effective, well-tolerated, and results in limited toxicity. As such, the general recommendation now is to use it as a second- or third-line therapy after surgery and SSAs. Sequencing of PRRT with respect to other currently available therapeutic options (including surgical debulking and interventional radiology/ liver directed therapies) has to do with the subtype, grade, SSTR expression, distribution, and bulk of disease. No clear guidelines or treatment algorithms exist with regard to PRRT as of yet, and there is no currently available prospective studies formally addressing PRRT sequencing, but significant research is ongoing. Ultimately, these therapeutic decisions should be made in the context of a multidisciplinary tumor board.

\section{Conflict of Interest Statement}

Dr. Rodney F. Pommier reports personal fees from Novartis Oncology, personal fees from Lexicon Pharmaceuticals, personal fees from Ipsen, and personal fees from Advanced Accelerator Applications, outside the submitted work. 
Dr. Erik S. Mittra reports grants from Advanced Accelerator Applications, personal fees from Advanced Accelerator Applications, personal fees from Advanced Accelerator Applications, personal fees from Curium Pharma, grants from ImaginAb, and grants from Nordic Nanovector, outside the submitted work.

Lauren M. Raymond, Tetiana Korzun, Adel Kardosh, and Kenneth J. Kolbeck have no relevant conflicts of interest to declare.

\section{Funding Sources}

No funding was received in the publication of this article.

\section{Author Contributions}

Lauren M. Raymond and Erik S. Mittra wrote the manuscript. Lauren M. Raymond and Tetiana Korzun performed the literature review including independently screening all titles, abstracts, and full texts for relevancy. Lauren M. Raymond, Tetiana Korzun, and Erik S. Mittra analyzed the literature with regard to peptide receptor radionuclide therapy and classified the available sequencing data and variables. Adel Kardosh, Kenneth J. Kolbeck, and Rodney F. Pommier provided critical revisions to the manuscript. All authors contributed to synthesis of the data and provided key information in the creation of this review.

\section{References}

1 Volante M, Brizzi MP, Faggiano A, La Rosa S, Rapa I, Ferrero A, et al. Somatostatin receptor type 2A immunohistochemistry in neuroendocrine tumors: a proposal of scoring system correlated with somatostatin receptor scintigraphy. Mod Pathol. 2007 Nov;20(11):117282.

2 Referenced with permission from the NCCN Clinical Practice Guidelines in Oncology (NCCN Guidelines ${ }^{\circledast}$ ) for Neuroendocrine and Adrenal Tumors V.1.2019. (C) National Comprehensive Cancer Network, Inc. 2019. All rights reserved. Accessed July 2, 2019. To view the most recent and complete version of the guideline, go online to NCCN.org. NCCN makes no warranties of any kind whatsoever regarding their content, use or application and disclaims any responsibility for their application or use in any way.

3 Bodei L, Mueller-Brand J, Baum RP, Pavel ME, Hörsch D, O’Dorisio MS, et al. The joint IAEA, EANM, and SNMMI practical guidance on peptide receptor radionuclide therapy (PRRNT) in neuroendocrine tumours. Eur J Nucl Med Mol Imaging. 2013 May;40(5): 800-16.

4 Kunz PL, Reidy-Lagunes D, Anthony LB, Bertino EM, Brendtro K, Chan JA, et al. Consensus guidelines for the management and treatment of neuroendocrine tumors. Pancreas. 2013 May;42(4):557-77.

5 Howe JR, Cardona K, Fraker DL, Kebebew E, Untch BR, Wang YZ, et al. The surgical management of small bowel neuroendocrine tumors: consensus guidelines of the North American Neuroendocrine Tumor Society. Pancreas. 2017 Jul;46(6):715-31.

6 Strosberg JR, Halfdanarson TR, Bellizzi AM, Chan JA, Dillon JS, Heaney AP, et al. The North American Neuroendocrine Tumor Society consensus guidelines for surveillance and medical management of midgut neuroendocrine tumors. Pancreas. 2017 Jul;46(6) 707-14.

7 Zandee WT, de Herder WW. The evolution of neuroendocrine tumor treatment reflected by ENETS guidelines. Neuroendocrinology. 2018;106(4):357-65.
8 Gonzalez-Flores E, Serrano R, Sevilla I, Viu$\operatorname{dez} \mathrm{A}$, Barriuso J, Benavent $\mathrm{M}$, et al. SEOM clinical guidelines for the diagnosis and treatment of gastroenteropancreatic and bronchial neuroendocrine neoplasms (NENs) (2018). Clin Transl Oncol. 2019 Jan;21(1):55-63.

9 Pavel M, Öberg K, Falconi M, Krenning EP, Sundin A, Perren A, et al. Gastroenteropancreatic neuroendocrine neoplasms: ESMO Clinical Practice Guidelines for diagnosis, treatment and follow-up. Ann Oncol. 2020 Jul;31(7):844-60.

10 Bergsland EK, Nakakura EK. Neuroendocrine tumors of unknown primary: is the primary site really not known? JAMA Surg. 2014 Sep;149(9):889-90.

11 Farley HA, Pommier RF. Surgical treatment of small bowel neuroendocrine tumors. Hematol Oncol Clin North Am. 2016 Feb;30(1):49-61.

12 Hellman P, Lundström T, Ohrvall U, Eriksson B, Skogseid B, Oberg K, et al. Effect of surgery on the outcome of midgut carcinoid disease with lymph node and liver metastases. World J Surg. 2002 Aug;26(8):991-7.

13 Ahmed A, Turner G, King B, Jones L, Culliford D, McCance D, et al. Midgut neuroendocrine tumours with liver metastases: results of the UKINETS study. Endocr Relat Cancer. 2009 Sep;16(3):885-94.

14 Lewis A, Raoof M, Ituarte PHG, Williams J, Melstrom L, Li D, et al. Resection of the primary gastrointestinal neuroendocrine tumor improves survival with or without liver treatment. Ann Surg. 2019 Dec;270(6):1131-7.

15 McEntee GP, Nagorney DM, Kvols LK, Moertel CG, Grant CS. Cytoreductive hepatic surgery for neuroendocrine tumors. Surgery. 1990 Dec;108(6):1091-6.

16 Sarmiento JM, Heywood G, Rubin J, Ilstrup DM, Nagorney DM, Que FG. Surgical treatment of neuroendocrine metastases to the liver: a plea for resection to increase survival. J Am Coll Surg. 2003 Jul;197(1):29-37.

17 Glazer ES, Tseng JF, Al-Refaie W, Solorzano CC, Liu P, Willborn KA, et al. Long-term survival after surgical management of neuroendocrine hepatic metastases. HPB. 2010 Aug; 12(6):427-33.
18 Mayo SC, de Jong MC, Pulitano C, Clary BM, Reddy SK, Gamblin TC, et al. Surgical management of hepatic neuroendocrine tumor metastasis: results from an international multi-institutional analysis. Ann Surg Oncol. 2010 Dec;17(12):3129-36.

19 Wiedenmann B, Jensen RT, Mignon M, Modlin CI, Skogseid B, Doherty G, et al. Preoperative diagnosis and surgical management of neuroendocrine gastroenteropancreatic tumors: general recommendations by a consensus workshop. World J Surg. 1998 Mar;22(3): 309-18.

20 Graff-Baker AN, Sauer DA, Pommier SJ, Pommier RF. Expanded criteria for carcinoid liver debulking: maintaining survival and increasing the number of eligible patients. Surgery. 2014 Dec;156(6):1369-7; discussion 767.

21 Morgan RE, Pommier SJ, Pommier RF. Expanded criteria for debulking of liver metastasis also apply to pancreatic neuroendocrine tumors. Surgery. 2018 Jan;163(1):218-25.

22 Maxwell JE, Sherman SK, O’Dorisio TM, Bellizzi AM, Howe JR. Liver-directed surgery of neuroendocrine metastases: what is the optimal strategy? Surgery. 2016 Jan;159(1):32033.

23 Howe JR, Merchant NB, Conrad C, Keutgen XM, Hallet J, Drebin JA, et al. The North American Neuroendocrine Tumor Society Consensus Paper on the surgical management of pancreatic neuroendocrine tumors. Pancreas. 2020 Jan;49(1):1-33.

24 Cives M, Ghayouri M, Morse B, Brelsford M, Black M, Rizzo A, et al. Analysis of potential response predictors to capecitabine/temozolomide in metastatic pancreatic neuroendocrine tumors. Endocr Relat Cancer. 2016 Sep;23(9):759-67.

25 Partelli S, Bertani E, Bartolomei M, Perali C, Muffatti F, Grana CM, et al. Peptide receptor radionuclide therapy as neoadjuvant therapy for resectable or potentially resectable pancreatic neuroendocrine neoplasms. Surgery. 2018 Apr;163(4):761-7. 
26 Madoff DC, Gupta S, Ahrar K, Murthy R, Yao JC. Update on the management of neuroendocrine hepatic metastases. J Vasc Interv Radiol. 2006 Aug; 17(8):1235-50; quiz 50.

27 Frilling A, Sotiropoulos GC, Li J, Kornasiewicz $\mathrm{O}$, Plöckinger U. Multimodal management of neuroendocrine liver metastases. HPB. 2010 Aug;12(6):361-79.

28 Harring TR, Nguyen NT, Goss JA, O’Mahony CA. Treatment of liver metastases in patients with neuroendocrine tumors: a comprehensive review. Int J Hepatol. 2011;2011:154541.

29 Chen JX, Wileyto EP, Soulen MC. Randomized Embolization Trial for NeuroEndocrine Tumor Metastases to the Liver (RETNET): study protocol for a randomized controlled trial. Trials. 2018 Jul 17;19(1):390.

30 Vogl TJ, Naguib NN, Zangos S, Eichler K, Hedayati A, Nour-Eldin NE. Liver metastases of neuroendocrine carcinomas: interventional treatment via transarterial embolization, chemoembolization and thermal ablation. Eur J Radiol. 2009 Dec;72(3):517-28.

31 Kolbeck KJ, Farsad K. Catheter-based treatments for hepatic metastases from neuroendocrine tumors. AJR Am J Roentgenol. 2014 Oct;203(4):717-24.

32 Riff BP, Yang YX, Soulen MC, Pryma DA, Bennett $B$, Wild $D$, et al. Peptide receptor radionuclide therapy-induced hepatotoxicity in patients with metastatic neuroendocrine tumors. Clin Nucl Med. 2015 Nov;40(11):845-50.

33 Susini C, Buscail L. Rationale for the use of somatostatin analogs as antitumor agents. Ann Oncol. 2006 Dec;17(12):1733-42.

34 Cakir M, Dworakowska D, Grossman A. Somatostatin receptor biology in neuroendocrine and pituitary tumours: part 2: clinical implications. J Cell Mol Med. 2010 Nov; 14(11):2585-91.

35 Rinke A, Müller HH, Schade-Brittinger C, Klose KJ, Barth P, Wied M, et al. Placebo-controlled, double-blind, prospective, randomized study on the effect of octreotide LAR in the control of tumor growth in patients with metastatic neuroendocrine midgut tumors: a report from the PROMID Study Group. J Clin Oncol. 2009 Oct 1;27(28):4656-63.

36 Caplin ME, Pavel M, Ćwikła JB, Phan AT, Raderer M, Sedláčková E, et al. Lanreotide in metastatic enteropancreatic neuroendocrine tumors. N Engl J Med. 2014 Jul 17;371(3): 224-33.

37 Wolin EM, Pavel M, Cwikla JB, Phan AT, Raderer M, Sedlackova E, et al. Final progression-free survival (PFS) analyses for lanreotide autogel/depot $120 \mathrm{mg}$ in metastatic enteropancreatic neuroendocrine tumors (NETs): the CLARINET extension study. J Clin Oncol. 2017;35(15 Suppl):4089-9.

38 O'Donnell A, Faivre S, Burris HA 3rd, Rea D, Papadimitrakopoulou V, Shand N, et al. Phase I pharmacokinetic and pharmacodynamic study of the oral mammalian target of rapamycin inhibitor everolimus in patients with advanced solid tumors. J Clin Oncol. 2008 Apr 1;26(10):1588-95.
39 Pavel ME, Hainsworth JD, Baudin E, Peeters M, Hörsch D, Winkler RE, et al. Everolimus plus octreotide long-acting repeatable for the treatment of advanced neuroendocrine tumours associated with carcinoid syndrome (RADIANT-2): a randomised, placebo-controlled, phase 3 study. Lancet. 2011 Dec 10; 378(9808):2005-12.

40 Yao JC, Shah MH, Ito T, Bohas CL, Wolin EM, Van Cutsem E, et al. Everolimus for advanced pancreatic neuroendocrine tumors. $\mathrm{N}$ Engl J Med. 2011 Feb 10;364(6):514-23.

41 Yao JC, Fazio N, Singh S, Buzzoni R, Carnaghi $\mathrm{C}$, Wolin E, et al. Everolimus for the treatment of advanced, non-functional neuroendocrine tumours of the lung or gastrointestinal tract (RADIANT-4): a randomised, placebo-controlled, phase 3 study. Lancet. 2016 Mar 5; 387(10022):968-77.

42 Raymond E, Dahan L, Raoul JL, Bang YJ, Borbath I, Lombard-Bohas C, et al. Sunitinib malate for the treatment of pancreatic neuroendocrine tumors. N Engl J Med. 2011 Feb 10; 364(6):501-13.

43 Garcia-Carbonero R, Sorbye H, Baudin E, Raymond E, Wiedenmann B, Niederle B, et al. ENETS consensus guidelines for high-grade gastroenteropancreatic neuroendocrine tumors and neuroendocrine carcinomas. Neuroendocrinology. 2016;103(2):186-94.

44 Sorbye H, Welin S, Langer SW, Vestermark LW, Holt N, Osterlund P, et al. Predictive and prognostic factors for treatment and survival in 305 patients with advanced gastrointestinal neuroendocrine carcinoma (WHO G3): the NORDIC NEC study. Ann Oncol. 2013 Jan; 24(1):152-60.

45 Yamaguchi T, Machida N, Morizane C, Kasuga A, Takahashi H, Sudo K, et al. Multicenter retrospective analysis of systemic chemotherapy for advanced neuroendocrine carcinoma of the digestive system. Cancer Sci. 2014 Sep;105(9):1176-81.

46 Clewemar Antonodimitrakis P, Sundin A, Wassberg C, Granberg D, Skogseid B, Eriksson B. Streptozocin and 5-fluorouracil for the treatment of pancreatic neuroendocrine tumors: efficacy, prognostic factors and toxicity. Neuroendocrinology. 2016;103(3-4):34553.

47 Kouvaraki MA, Ajani JA, Hoff P, Wolff R, Evans DB, Lozano R, et al. Fluorouracil, doxorubicin, and streptozocin in the treatment of patients with locally advanced and metastatic pancreatic endocrine carcinomas. J Clin Oncol. 2004 Dec 1;22(23):4762-71.

48 Strosberg JR, Fine RL, Choi J, Nasir A, Coppola D, Chen DT, et al. First-line chemotherapy with capecitabine and temozolomide in patients with metastatic pancreatic endocrine carcinomas. Cancer. 2011 Jan 15;117(2):26875 .

49 Reubi JC. Peptide receptors as molecular targets for cancer diagnosis and therapy. Endocr Rev. 2003 Aug;24(4):389-427.
50 Seregni E, Maccauro M, Coliva A, Castellani MR, Bajetta E, Aliberti G, et al. Treatment with tandem [(90)Y]DOTA-TATE and [(177) $\mathrm{Lu}$ ] DOTA-TATE of neuroendocrine tumors refractory to conventional therapy: preliminary results. Q J Nucl Med Mol Imaging. 2010 Feb;54(1):84-91.

51 Anthony LB, Woltering EA, Espenan GD Cronin MD, Maloney TJ, McCarthy KE. Indium-111-pentetreotide prolongs survival in gastroenteropancreatic malignancies. Semin Nucl Med. 2002 Apr;32(2):123-32.

52 Kwekkeboom DJ, Bakker WH, Kam BL, Teunissen JJ, Kooij PP, de Herder WW, et al. Treatment of patients with gastro-enteropancreatic (GEP) tumours with the novel radiolabelled somatostatin analogue $[177 \mathrm{Lu}-$ DOTA(0),Tyr3] octreotate. Eur J Nucl Med Mol Imaging. 2003 Mar;30(3):417-22.

53 Nguyen C, Faraggi M, Giraudet AL, de Labriolle-Vaylet C, Aparicio T, Rouzet F, et al. Long-term efficacy of radionuclide therapy in patients with disseminated neuroendocrine tumors uncontrolled by conventional therapy. J Nucl Med. 2004 Oct;45(10):1660-8.

54 Bushnell DL Jr, O’Dorisio TM, O’Dorisio MS Menda Y, Hicks RJ, Van Cutsem E, et al. 90Yedotreotide for metastatic carcinoid refractory to octreotide. J Clin Oncol. 2010 Apr 1; 28(10):1652-9.

55 Baum RP, Kluge AW, Kulkarni H, SchorrNeufing U, Niepsch K, Bitterlich N, et al. [(177)Lu-DOTA](0)-D-Phe(1)-Tyr(3)-Octreotide ((177)Lu-DOTATOC) for peptide receptor radiotherapy in patients with advanced neuroendocrine tumours: a phase-II study. Theranostics. 2016;6(4):501-10.

56 Katona BW, Roccaro GA, Soulen MC, Yang YX, Bennett BJ, Riff BP, et al. Efficacy of peptide receptor radionuclide therapy in a United States-based cohort of metastatic neuroendocrine tumor patients: single-institution retrospective analysis. Pancreas. 2017 Oct;46(9): 1121-6.

57 Strosberg J, El-Haddad G, Wolin E, Hendifar A, Yao J, Chasen B, et al. Phase 3 trial of (177) Lu-dotatate for midgut neuroendocrine tumors. N Engl J Med. 2017 Jan 12;376(2):12535.

58 Lutathera (lutetium Lu 177 dotatate) injection, for intravenous use [prescribing information]. Millburn, NJ: Advanced Accelerator Applications; $2018 \mathrm{Jul}$

59 Valkema R, Pauwels SA, Kvols LK, Kwekkeboom DJ, Jamar F, de Jong M, et al. Long-term follow-up of renal function after peptide receptor radiation therapy with (90)YDOTA(0), Tyr(3)-octreotide and (177)Lu$\operatorname{DOTA}(0), \operatorname{Tyr}(3)$-octreotate. J Nucl Med. 2005 Jan;46(Suppl 1):83S-91S.

60 Imhof A, Brunner P, Marincek N, Briel M, Schindler C, Rasch H, et al. Response, surviv$\mathrm{al}$, and long-term toxicity after therapy with the radiolabeled somatostatin analogue [90YDOTA]-TOC in metastasized neuroendocrine cancers. J Clin Oncol. 2011 Jun 10; 29(17):2416-23. 
61 Lapa C, Werner RA, Bluemel C, Lückerath K, Schirbel A, Strate A, et al. Influence of the amount of co-infused amino acids on posttherapeutic potassium levels in peptide receptor radionuclide therapy. EJNMMI Res. 2014; 4:46.

62 Behr TM, Goldenberg DM, Becker W. Reducing the renal uptake of radiolabeled antibody fragments and peptides for diagnosis and therapy: present status, future prospects and limitations. Eur J Nucl Med. 1998 Feb;25(2): 201-12.

63 Jamar F, Barone R, Mathieu I, Walrand S, Labar D, Carlier P, et al. 86Y-DOTA0)-D-Phe1Tyr3-octreotide (SMT487): a phase 1 clinical study: pharmacokinetics, biodistribution and renal protective effect of different regimens of amino acid co-infusion. Eur J Nucl Med Mol Imaging. 2003 Apr;30(4):510-8.

64 Kwekkeboom DJ, Mueller-Brand J, Paganelli G, Anthony LB, Pauwels S, Kvols LK, et al. Overview of results of peptide receptor radionuclide therapy with 3 radiolabeled somatostatin analogs. J Nucl Med. 2005 Jan; 46(Suppl 1):62S-6S.

65 Kalshetty A, Ramaswamy A, Ostwal V, Basu S. Resistant functioning and/or progressive symptomatic metastatic gastroenteropancreatic neuroendocrine tumors: efficacy of 177Lu-DOTATATE peptide receptor radionuclide therapy in this setting. Nucl Med Commun. 2018 Dec;39(12):1143-9.

66 Sowa-Staszczak A, Pach D, Chrzan R, Trofimiuk M, Stefańska A, Tomaszuk M, et al. Peptide receptor radionuclide therapy as a potential tool for neoadjuvant therapy in patients with inoperable neuroendocrine tumours (NETs). Eur J Nucl Med Mol Imaging. 2011 Sep;38(9):1669-74.

67 Yordanova A, Wicharz MM, Mayer K, Brossart P, Gonzalez-Carmona MA, Strassburg $\mathrm{CP}$, et al. The role of adding somatostatin analogues to peptide receptor radionuclide therapy as a combination and maintenance therapy. Clin Cancer Res. 2018 Oct 1;24(19): $4672-9$.

68 Biliotti G, Martini F, Vaggelli L, Messerini L, Colagrande S, Pupi A, et al. Multiple effects of somatostatin analogs verified in three cases of metastasized neuroendocrine tumors of the gastroenteropancreatic system. Tumori. 2006 Mar-Apr;92(2):170-4.
69 Claringbold PG, Price RA, Turner JH. Phase I-II study of radiopeptide $177 \mathrm{Lu}$-octreotate in combination with capecitabine and temozolomide in advanced low-grade neuroendocrine tumors. Cancer Biother Radiopharm. 2012 Nov;27(9):561-9.

70 ClinicalTrials.gov [Internet]. Treatment of neuroendocrine tumors (NETs) with combination of everolimus and radiolabeled somatostatin analogue. Bethesda, MD: National Library of Medicine; 2018 Aug 14. Identifier NCT03629847. Available from: https://clinicaltrials.gov/ct2/show/NCT03629847.

71 Makis W, McCann K, Buteau FA, McEwan AJ. Resolution of malignant ascites and stabilization of metastases in a patient with small bowel neuroendocrine tumor with $177 \mathrm{Lu}$ DOTATATE following progression after 17 131I-MIBG treatments and chemotherapy. Clin Nucl Med. 2015 Jul;40(7):564-6.

72 Severi S, Sansovini M, Ianniello A, Bodei L, Nicolini S, Ibrahim T, et al. Feasibility and utility of re-treatment with (177)Lu-DOTATATE in GEP-NENs relapsed after treatment with (90)Y-DOTATOC. Eur J Nucl Med Mol Imaging. 2015 Dec;42(13):1955-63.

73 van der Zwan WA, Brabander T, Kam BLR Teunissen JJM, Feelders RA, Hofland J, et al. Salvage peptide receptor radionuclide therapy with [(177)Lu-DOTA,Tyr(3)] octreotate in patients with bronchial and gastroenteropancreatic neuroendocrine tumours. Eur J Nucl Med Mol Imaging. 2019 Mar;46(3):704-17.

74 Faggiano A, Di Maio S, Mocerino C, Ottaviano M, De Divitiis C, Guarnotta V, et al. Therapeutic sequences in patients with grade 1-2 neuroendocrine tumors (NET): an observational multicenter study from the ELIOS group. Endocrine. 2019 Nov;66(2):417-24.

75 Genc CG, Klümpen HJ, van Oijen MGH, van Eijck CHJ, Nieveen van Dijkum EJM. A nationwide population-based study on the survival of patients with pancreatic neuroendocrine tumors in The Netherlands. World J Surg. 2018 Feb;42(2):490-7.

76 ClinicalTrials.gov [Internet]. Efficacy and Safety of $177 \mathrm{Lu}$-edotreotide PRRT in GEPNET Patients (COMPETE). Bethesda, MD: National Library of Medicine; 2017 Feb 9. Identifier NCT03049189. Available from: https://clinicaltrials.gov/ct2/show/ NCT03049189.
77 ClinicalTrials.gov [Internet]. Antitumor Efficacy of Peptide Receptor Radionuclide Therapy With 177Lutetium -Octreotate Randomized vs Sunitinib in Unresectable Progressive Well-differentiated Neuroendocrine Pancreatic Tumor: First Randomized Phase II (OCCLURANDOM). Bethesda, MD: National Library of Medicine; 2014 Sep 3. Identifier NCT02230176. Available from: https://clinicaltrials.gov/ct2/show/NCT02230176.

78 ClinicalTrials.gov [Internet]. Randomized Phase III of PRRT Versus Interferon (CASTOR). Bethesda, MD: National Library of Medicine; 2013 May 23. Identifier NCT01860742. Available from: https://clinicaltrials.gov/ct2/show/NCT01860742.

79 ClinicalTrials.gov [Internet]. Pembrolizumab in With Liver-Directed or Peptide Receptor Radionuclide Therapy in Neuroendocrine Tumors With Metastases. Bethesda, MD: National Library of Medicine; 2019 Mar 8. Identifier NCT03457948. Available from: https:// clinicaltrials.gov/ct2/show/NCT03457948.

80 ClinicalTrials.gov [Internet]. Capecitabine ON Temozolomide Radionuclide Therapy Octreotate Lutetium-177 NeuroEndocrine Tumours Study (CONTROL NETS). Bethesda, MD: National Library of Medicine; 2015 Feb 9. Identifier NCT02358356. Available from: https: //clinicaltrials.gov/ct2/show/ NCT02358356.

81 ClinicalTrials.gov [Internet]. Personalized CAPTEM Radiopeptide Therapy of Advanced, Non-resectable Neuroendocrine Cancer. Bethesda, MD: National Library of Medicine; 2019 Dec 11. Identifier NCT04194125. Available from: https://clinicaltrials.gov/ct2/show/NCT04194125.

82 ClinicalTrials.gov [Internet]. 177Lutethium: Peptide Receptor Radionuclide Therapy (LuPRRT) Plus Capecitabine Versus Lu-PRRT in FDG Positive, Gastro-entero-pancreatic Neuroendocrine Tumors (Lu-Ca-S). Bethesda, MD: National Library of Medicine; 2016 Apr 13. Identifier NCT02736448. Available from:

83 ClinicalTrials.gov [Internet]. Study to Evaluate the Efficacy and Safety of Lutathera in $\mathrm{Pa}$ tients With Grade 2 and Grade 3 Advanced GEP-NET (NETTER-2). Bethesda, MD: National Library of Medicine; 2019 Jun 3. Identifier NCT03972488. Available from: https:// clinicaltrials.gov/ct2/show/NCT03972488. 